\title{
Effect of the angiotensin II receptor blocker valsartan on cardiac hypertrophy and myocardial histone deacetylase expression in rats with aortic constriction
}

\author{
WEI-PING XU ${ }^{1 *}$, TONG-QING YAO ${ }^{1 *}$, YI-BO JIANG ${ }^{2}$, MAO-ZHEN ZHANG $^{1}$, \\ YUE-PENG WANG ${ }^{1}$, YING YU ${ }^{1}$, JING-XIANG LI ${ }^{1}$ and YI-GANG LI ${ }^{1}$ \\ ${ }^{1}$ Department of Cardiology, Xin Hua Hospital Affiliated to Shanghai Jiao Tong University School of Medicine, \\ Shanghai 200092; ${ }^{2}$ Department of Cardiology, Taixing People's Hospital, Taixing, Jiangsu 225400, P.R. China
}

Received April 24, 2014; Accepted November 11, 2014

DOI: $10.3892 /$ etm.2015.2374

\begin{abstract}
The aim of the present study was to observe the myocardial expression of members of the histone deacetylase (HDAC) family (HDAC2, HDAC5 and HDAC9) in rats with or without myocardial hypertrophy $(\mathrm{MH})$ in the presence and absence of the angiotensin II receptor blocker valsartan. Adult male Wistar rats were randomly divided into three groups ( $n=6 /$ group): Sham-operated control rats, treated with distilled water $(1 \mathrm{ml} /$ day) through gavage; rats with $\mathrm{MH}$ (established through aortic constriction), treated with distilled water (1 $\mathrm{ml} /$ day) through gavage; and $\mathrm{MH}+$ valsartan rats, treated with $20 \mathrm{mg} / \mathrm{kg} /$ day valsartan through gavage. Treatments commenced one day after surgery and continued for eight weeks. Body weight (BW), heart weight (HW) and plasma atrial natriuretic peptide (ANP) and brain natriuretic peptide (BNP) levels were determined, and the myocardial expression of HDAC2, HDAC5 and HDAC9 was analyzed through a reverse transcription semi-quantitative polymerase chain reaction. The BWs of the rats in the three groups were similar at baseline; however, after eight weeks the $\mathrm{BW}$ of the rats in the $\mathrm{MH}+$ valsartan group was significantly reduced compared with that of the $\mathrm{MH}$ rats. Furthermore, the $\mathrm{HW} / \mathrm{BW}$ ratio and plasma ANP and BNP levels were increased, the myocardial HDAC2 expression was significantly upregulated and the HDAC5 and HDAC9 expression was significantly downregulated in the $\mathrm{MH}$ rats compared with those in the control rats; however, these changes were significantly attenuated by valsartan. Modulation of myocardial
\end{abstract}

Correspondence to: Professor Yi-Gang Li, Department of Cardiology, Xin Hua Hospital Affiliated to Shanghai Jiao Tong University School of Medicine, 1665 Kongjiang Road, Shanghai 200092, P.R. China

E-mail: doctorliyig@126.com

*Contributed equally

Key words: histone deacetylases, angiotensin II receptor blockers, cardiac hypertrophy
HDAC5, HDAC9 and HDAC2 expression may therefore be one of the anti-hypertrophic mechanisms of valsartan in this rat $\mathrm{MH}$ model.

\section{Introduction}

Myocardial hypertrophy $(\mathrm{MH})$ is involved in the pathogenesis of various cardiovascular diseases (1-3); however, the molecular mechanism underlying $\mathrm{MH}$ is yet to be fully understood. It has been shown that dysregulation of gene transcription can promote $\mathrm{MH}$ and cardiac dysfunction (4). The acetylation and deacetylation of core histones via histone acetyltransferases and histone deacetylases (HDACs) are important regulatory mechanisms in the pathogenesis of MH (5). A previous study reported the presence of 18 different HDACs, divided in two families: The first family includes HDACs belonging to Classes I (HDAC1, HDAC2, HDAC3 and HDAC8), II (HDAC4, HDAC5, HDAC6, HDAC7, HDAC9 and HDAC10) and IV (HDAC11); the second family of HDACs comprises seven members belonging to the Class III HDACs or sirtuins (SIRT1-7) (6).

Among the HDACs, HDAC2, HDAC5 and HDAC9 are the major players actively involved in regulating the processes of $\mathrm{MH}$ (7-9). It has been shown that the nonspecific HDAC inhibitors trichostatin $\mathrm{A}$ and valproic acid, as well as the HDAC-selective inhibitor SK-7041, can attenuate angiotensin II- and aortic stenosis-induced MH (10). Furthermore, experimental and clinical studies have demonstrated the beneficial effects of certain angiotensin II receptor blockers (ARBs) on inhibiting and attenuating MH (11-13). Despite this, it has yet to be determined whether the anti-hypertrophic effects of ARBs are partially mediated by modulating the myocardial expression of HDAC2, HDAC5 and HDAC9. In the present study, the association between myocardial HDAC2, HDAC5 and HDAC9 expression and $\mathrm{MH}$ was observed in rats with aortic constriction (AC) and/or ARB blocker (valsartan) treatment.

\section{Materials and methods}

Ethics statement. All animal protocols in this study were approved by the Animal Care and Use Committee of the Research Institute of Medicine, Shanghai Jiao Tong University 
Table I. BW and HW of rats eight weeks after aortic constriction surgery.

\begin{tabular}{lccc}
\hline & Control group & MH group & Valsartan group \\
\hline BW (g) & $262.4 \pm 8.0$ & $257.3 \pm 10.1$ & $241.7 \pm 10.1^{\mathrm{a}}$ \\
HW (mg) & $170.2 \pm 10.6$ & $260.3 \pm 20.1^{\mathrm{a}}$ & $200.2 \pm 11.7^{\mathrm{a}, \mathrm{b}}$ \\
HW/BW & $0.65 \pm 0.10$ & $1.09 \pm 0.05^{\mathrm{a}}$ & $0.83 \pm 0.08^{\mathrm{b}}$ \\
\hline
\end{tabular}

${ }^{a} \mathrm{P}<0.05$ compared with the control group; ${ }^{b} \mathrm{P}<0.05$ compared with the $\mathrm{MH}$ group. $\mathrm{MH}$, myocardial hypertrophy; BW, body weight; HW, heart weight.

(Shanghai, China), in accordance with National Institutes of Health guidelines and public law. All surgery was performed under sodium pentobarbital anesthesia, and all efforts were made to minimize suffering.

In vivo hypertrophy models and blood pressure (BP) measurements. Five- to eight-week-old adult male Wistar rats were purchased from the Shanghai SLAC Laboratory Animal Co., Ltd. (Chinese Academy of Sciences, Shanghai, China) and housed individually in plastic cages in a temperature-controlled room. Rats were randomly divided into sham-operated control, $\mathrm{MH}$ and $\mathrm{MH}+$ valsartan groups (n=6/group). $\mathrm{MH}$ was induced by abdominal aortic banding as previously described (14). Briefly, the animals were anesthetized with ketamine (16.65 mg/kg intramuscularly), a 22-gauge needle was placed along the abdominal aorta above the renal arteries and both the aorta and the needle were tied with a 7-0 silk thread. The needle was removed, leaving an aortic lumen determined by the diameter of the needle (60-65\% stenosis). The sham-operated animals were subjected to the same procedure without the aortic banding. One day post-surgery, valsartan (20 mg/kg; Beijing Novartis Pharma Co., Ltd., Beijing, China) was administered to rats in the $\mathrm{MH}+$ valsartan group through gavage once daily for eight weeks. The sham-operated animals and rats in the $\mathrm{MH}$ group received $1 \mathrm{ml}$ distilled water through gavage daily for eight weeks. The heart weight to body weight (HW/BW) ratio was obtained and systolic BP measurements were made using tail-cuff plethysmography (15) prior to surgery and every two weeks after the surgery; mean values from three measurements at each time-point were calculated.

Histology. Cross-sectional areas of cardiomyocytes and myocardial morphological changes were observed under optical microscope on hematoxylin and eosin-stained sections by an investigator blinded to the study design.

Measurements of plasma atrial natriuretic peptide (ANP) and brain natriuretic peptide (BNP) levels and myocardial HDAC2, HDAC5 and HDAC9 mRNA expression. Plasma ANP and BNP levels were determined with immunoradiometric assay as previously described (16). Myocardial HDAC2, HDAC5 and HDAC9 mRNA expression was detected through a reverse transcription semi-quantitative polymerase chain reaction (RT-qPCR) method. Total RNA was extracted from the myocardial tissue with TRIzol ${ }^{\circledR}$ (Invitrogen Life Technologies, Carlsbad, CA, USA). RT was carried out with $1.0 \mathrm{~g}$ total RNA using the SuperScript ${ }^{\circledR}$ First-Strand

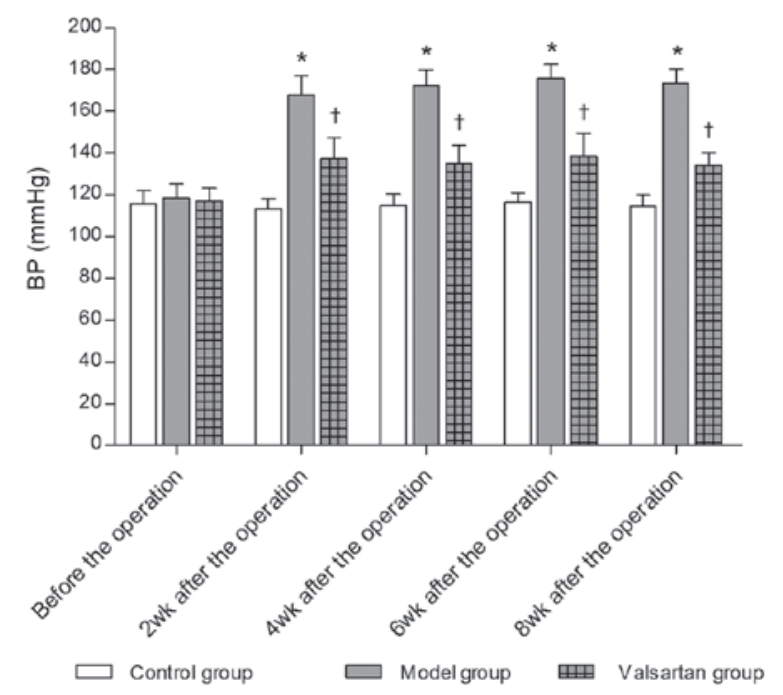

Figure 1. Time course of BP changes in the three groups. Systolic BP increased significantly in the $\mathrm{MH}$ rats, and this increase was partly attenuated by valsartan. Results are presented as the mean \pm standard deviation. ${ }^{*} \mathrm{P}<0.05$ compared with the control group; ${ }^{\dagger} \mathrm{P}<0.05$ compared with the $\mathrm{MH}$ group. $\mathrm{BP}$ blood pressure; MH, myocardial hypertrophy; wk, weeks.

Synthesis System for RT-PCR (Invitrogen Life Technologies), according to manufacturer's instructions. cDNA (10 ng) was subjected to semi-quantitative PCR using TaqMan ${ }^{\circledR}$ gene expression assays (Applied Biosystems, Foster City, CA, USA) to assess the expression level of HDAC2 (cat.no.Mm01193631_m1),HDAC5(cat.no.Mm00515917_m1), and HDAC9 (cat. no. Mm00458456_m1). The semi-quantitative PCR cycling conditions were as follows: $94^{\circ} \mathrm{C}$ for $1 \mathrm{~min}$, $55^{\circ} \mathrm{C}$ for $1 \mathrm{~min}$ and $72^{\circ} \mathrm{C}$ for $1 \mathrm{~min}$ for 35 cycles, followed by $72^{\circ} \mathrm{C}$ for $5 \mathrm{~min}$. Quantity One V4.62 (Bio-Rad, Hercules, CA, USA) software was used to analyse the results of the gray values from the semi-quantitative PCR.

Statistical analysis. Results are expressed as the mean \pm standard deviation and analyzed using one-way analysis of variance with Bonferroni post hoc comparison analysis. $\mathrm{P}<0.05$ was considered to indicate a statistically significant difference.

\section{Results}

Myocardial hypertrophy. Eight weeks after surgery, the BW of the rats in the $\mathrm{MH}+$ valsartan group was significantly reduced compared with that in the $\mathrm{MH}$ rats. As expected, the 
Table II. Plasma ANP and BNP levels.

\begin{tabular}{lccc}
\hline & Control group & MH group & Valsartan group \\
\hline ANP $(\mathrm{pg} / \mathrm{ml})$ & $53.1 \pm 11.3$ & $90.8 \pm 7.8^{\mathrm{a}}$ & $60.1 \pm 17.7^{\mathrm{b}}$ \\
BNP $(\mathrm{pg} / \mathrm{ml})$ & $136.6 \pm 11.2$ & $174.4 \pm 43.8^{\mathrm{a}}$ & $121.5 \pm 20.8^{\mathrm{b}}$ \\
\hline
\end{tabular}

${ }^{\mathrm{a}} \mathrm{P}<0.05$ compared with the control group; ${ }^{\mathrm{b}} \mathrm{P}<0.05$ compared with the $\mathrm{MH}$ group. $\mathrm{MH}$, myocardial hypertrophy; ANP, atrial natriuretic peptide; BNP, brain natriuretic peptide.

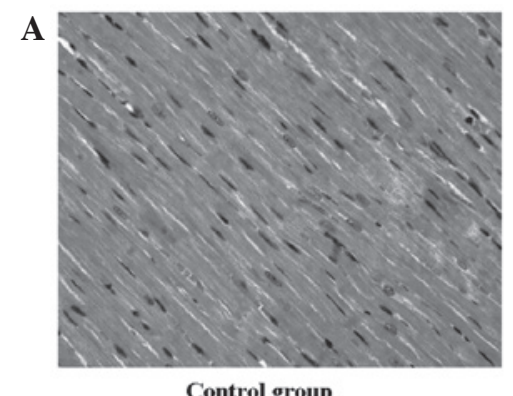

Control group
B

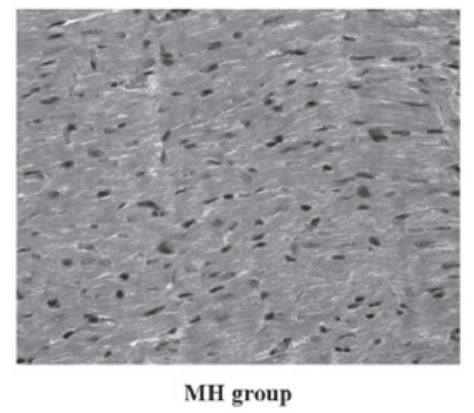

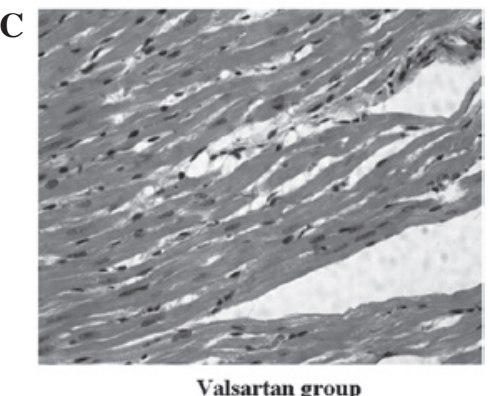

Valsartan group

Figure 2. Hematoxylin and eosin staining of the myocardium in the (A) control, (B) MH and (C) MH + valsartan groups (magnification, $\mathrm{x} 400$ ). (A) Eight weeks post-aortic constriction, the left ventricular cardiomyocytes in the control group were observed to form a regular array, with uniform size and non-swollen nuclei. (B) By contrast, cardiomyocytes in the MH rats formed a disorderly, irregular array, with non-uniform sizes and swollen nuclei; (C) these changes were partially reversed by valsartan. $\mathrm{MH}$, myocardial hypertrophy.
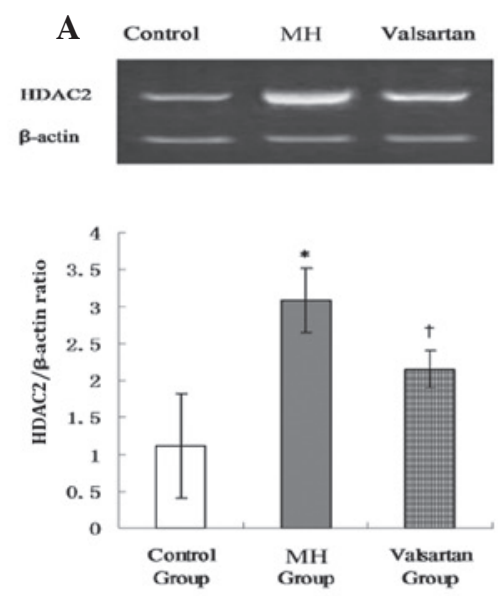
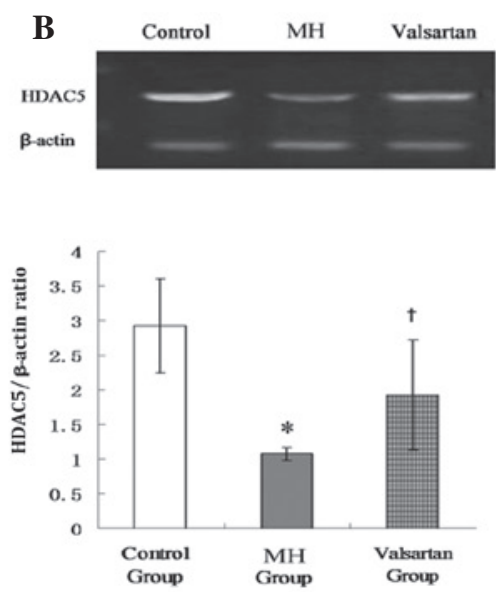
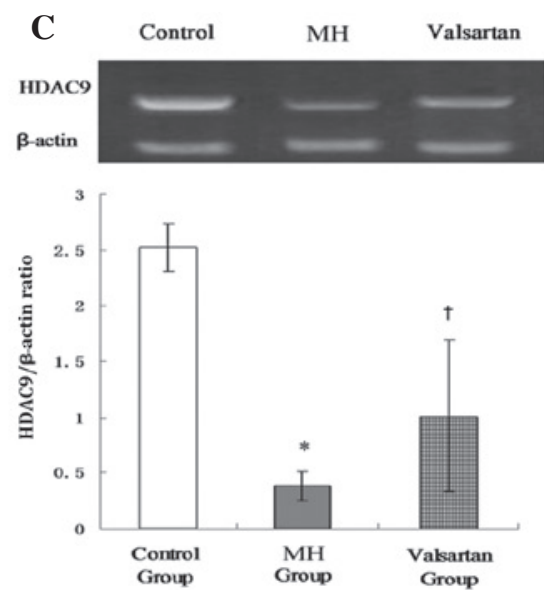

Figure 3. Myocardial (A) HDAC2, (B) HDAC5 and (C) HDAC9 expression in the three groups eight weeks after surgery was detected through a reverse transcription semi-quantitative polymerase chain reaction. The myocardial expression of HDAC2 was significantly upregulated while the myocardial expression of HDAC5 and HDAC9 was significantly downregulated in the MH hearts compared with expression in the control group; however, these changes could be partially reversed by valsartan. Results are presented as the mean \pm standard deviation. ${ }^{*} \mathrm{P}<0.05$ compared with the control group; ${ }^{\dagger} \mathrm{P}<0.05$ compared with the MH group. MH, myocardial hypertrophy; HDAC, histone deacetylase.

HW and HW/BW were significantly increased in the MH rats compared with those in the control rats, and could be significantly reduced by valsartan treatment (Table I).

Blood pressure. As shown in Fig. 1, systolic BP was significantly increased in the $\mathrm{MH}$ rats compared with that in the control rats, but was normalized with valsartan.

Histology. Histological observations were performed eight weeks post-AC. Compared with the control group (Fig. 2A), moderate hypertrophy of the left ventricular cardiomyocytes and enlarged nuclei were observed in $\mathrm{MH}$ hearts (Fig. 2B).
Only mild hypertrophy of the cardiomyocytes could be observed following valsartan treatment (Fig. 2C).

Plasma ANP and BNP levels. Plasma ANP and BNP levels were significantly increased in the $\mathrm{MH}$ rats compared with those in the sham-operated rats eight weeks after surgery. Valsartan treatment significantly reduced the ANP and BNP levels (Table II).

Myocardial mRNA expression of HDAC2, HDAC5 and $H D A C 9$. Eight weeks post-AC, the myocardial expression of HDAC2 was significantly upregulated and the myocar- 
dial expression of HDAC5 and HDAC9 was significantly downregulated in the $\mathrm{MH}$ hearts compared with that in the sham-operated hearts. These changes could be reversed by valsartan (Fig. 3A-C).

\section{Discussion}

In the present study, it was shown that $\mathrm{AC}$-induced $\mathrm{MH}$ was associated with myocardial HDAC expression changes: HDAC2 was upregulated while HDAC5 and HDAC9 were downregulated in MH hearts. These changes could be reversed by valsartan, suggesting that the anti-hypertrophic effects of valsartan could be partly associated with the changes in myocardial HDAC expression. To the best of our knowledge, that is the first report concerning the effects of valsartan on myocardial HDAC expression in an AC-induced model of MH.

It has previously been shown that the dysregulation of gene transcription can promote cardiomyocyte hypertrophy and embryonic gene expression and thus influence cardiac function (17). Furthermore, it has been shown that the enzymes controlling histone acetylation may serve as stress regulators in gene expression in the heart (18), and histone acetylation/deacetylation may be a focal point for the control of cardiac growth and gene expression in response to acute and chronic stress stimuli (19).

The present results have shown that HDAC2 is upregulated in $\mathrm{MH}$ rats. It is known that HDAC2 regulates the expression of numerous fetal cardiac isoforms. HDAC2 deficiency or chemical HDAC2 inhibition can prevent the re-expression of fetal genes and attenuate cardiac hypertrophy in hearts exposed to hypertrophic stimuli (20). The present finding that upregulated HDAC2 expression in AC-induced MH hearts can be partly reversed by valsartan is consistent with the above results and suggests that one of the anti-hypertrophic mechanisms of valsartan may be associated with the modulatory effect of valsartan on myocardial HDAC2 expression.

In contrast to HDAC2, HDAC5 and HDAC9 are hypertrophy suppressors, and mice lacking HDAC5 (20) or HDAC9 (21) have been shown to be prone to hypertrophic stimuli. Consistent with the above results, the present study found downregulated HDAC5 and HDAC9 myocardial expression in MH hearts compared with the control hearts; however, the myocardial HDAC5 and HDAC9 expression was significantly upregulated following valsartan treatment.

Increases in BP and plasma ANP and BNP levels are typical findings an $\mathrm{AC}$ rat model $(22,23)$. As expected, valsartan reduced the BP and plasma ANP and BNP levels, indicating that $\mathrm{BP}$ reduction serves as an important mechanism in the attenuation of $\mathrm{AC}$-induced $\mathrm{MH}$ and that reduced plasma ANP and BNP levels may be the consequence of reduced $\mathrm{MH}$ following valsartan therapy.

In conclusion, the anti-hypertrophic effects of valsartan may be partially mediated by changes in myocardial HDAC5, HDAC9 and HDAC2 expression in this AC rat model. Further studies in animals with silenced or overexpressed HDAC5, HDAC9 and HDAC2 gene expression are required to establish the role of HDAC5, HDAC9 and HDAC2 in the valsartan-induced effects on $\mathrm{MH}$.

\section{Acknowledgements}

This study was supported by grants from the National Natural Science Foundation of China (nos. 30670831 and 30871082).

\section{References}

1. Zhang CL, McKinsey TA, Chang S, et al: Class II histone deacetylases act as signal-responsive repressors of cardiac hypertrophy. Cell 110: 479-488, 2002.

2. Berridge MJ: Remodelling $\mathrm{Ca}^{2+}$ signalling systems and cardiac hypertrophy. Biochem Soc Trans 34: 228-231, 2006.

3. Bisping E, Ikeda S, et al: Transcription factor GATA4 is activated but not required for insulin-like growth factor 1 (IGF1)-induced cardiac hypertrophy. J Biol Chem 287: 9827-9834, 2012.

4. Toko H, Minamino T and Komuro I: Role of heat shock transcriptional factor 1 and heat shock proteins in cardiac hypertrophy. Trends Cardiovasc Med 18: 88-93, 2008.

5. Xie M and Hill JA: HDAC-dependent ventricular remodeling. Trends Cardiovasc Med 23: 229-235, 2013.

6. Di Marcotullio L, Canettieri G, Infante P, et al: Protected from the inside: endogenous histone deacetylase inhibitors and the road to cancer. Biochim Biophys Acta 1815: 241-252, 2011.

7. Agalioti T, Chen G and Thanos D: Deciphering the transcriptional histone acetylation code for a human gene. Cell 111: 381-392, 2002.

8. Hang CT, Yang J, Han P, et al: Chromatin regulation by Brg1 underlies heart muscle development and disease. Nature 466: 62-67, 2010.

9. Pedram A, Razandi M, Narayanan R, et al: Estrogen regulates histone deacetylases to prevent cardiac hypertrophy. Mol Biol Cell 24: 3805-3818, 2013.

10. Kee HJ, Sohn IS, Nam KI, et al: Inhibition of histone deacetylation blocks cardiac hypertrophy induced by angiotensin II infusion and aortic banding. Circulation 113: 51-59, 2006.

11. Prisant LM: Management of hypertension in patients with cardiac disease: use of renin-angiotensin blocking agents. Am J Med 121 (8 Suppl): S8-S15, 2008.

12. Shimada YJ, Passeri JJ, Baggish AL, et al: Effects of losartan on left ventricular hypertrophy and fibrosis in patients with nonobstructive hypertrophic cardiomyopathy. JACC Heart Fail 1: 480-487, 2013.

13. Li L, Zhou N, Gong H, et al: Comparison of angiotensin II type 1-receptor blockers to regress pressure overload-induced cardiac hypertrophy in mice. Hypertens Res 33: 1289-1297, 2010.

14. Gao S, Long CL, Wang RH, et al: K(ATP) activation prevents progression of cardiac hypertrophy to failure induced by pressure overload via protecting endothelial function. Cardiovasc Res 83: 444-456, 2009.

15. Lovenberg W: Animal models for hypertension research. Prog Clin Biol Res 229: 225-240, 1987.

16. Del Ry S, Clerico A, Giannessi D, et al: Measurement of brain natriuretic peptide in plasma samples and cardiac tissue extracts by means of an immunoradiometric assay method. Scand J Clin Lab Invest 60: 81-90, 2000.

17. Wang N, Frank GD, Ding R, et al: Promyelocytic leukemia zinc finger protein activates GATA4 transcription and mediates cardiac hypertrophic signaling from angiotensin II receptor 2. PLoS One 7: e35632, 2012.

18. Colussi C, Illi B, Rosati J, et al: Histone deacetylase inhibitors: keeping momentum for neuromuscular and cardiovascular diseases treatment. Pharmacol Res 62: 3-10, 2010.

19. Chu CH, Lo JF, Hu WS, et al: Histone acetylation is essential for ANG-II-induced IGF-IIR gene expression in H9c2 cardiomyoblast cells and pathologically hypertensive rat heart. J Cell Physiol 227: 259-268, 2012.

20. Eom GH, Nam YS, Oh JG, et al: Regulation of acetylation of histone deacetylase 2 by $\mathrm{p} 300 / \mathrm{CBP}$-associated factor/histone deacetylase 5 in the development of cardiac hypertrophy. Circ Res 114: 1133-1143, 2014.

21. Greco TM, Yu F, Guise AJ and Cristea IM: Nuclear import of histone deacetylase 5 by requisite nuclear localization signal phosphorylation. Mol Cell Proteomics 10: M110.004317, 2011.

22. Nader L, Lahoud L, Chouery E, et al: B-type natriuretic peptide receptors in hypertrophied adult rat cardiomyocytes. Ann Cardiol Angeiol (Paris) 59: 20-24, 2010.

23. Ito H, Hiroe M, Hirata Y, et al: Endothelin ETA receptor antagonist blocks cardiac hypertrophy provoked by hemodynamic overload 89: 2198-2203, 1994. 